\title{
Ring coproducts embedded in power-series rings
}

\author{
Pere Ara and Warren Dicks
}

June 8, 2021

\begin{abstract}
Let $R$ be a ring (associative, with 1 ), and let $R\langle\langle a, b\rangle\rangle$ denote the powerseries $R$-ring in two non-commuting, $R$-centralizing variables, $a$ and $b$. Let $A$ be an $R$-subring of $R\langle\langle a\rangle\rangle$ and $B$ be an $R$-subring of $R\langle\langle b\rangle\rangle$, and let $\alpha$ denote the natural map $A \amalg_{R} B \rightarrow R\langle\langle a, b\rangle\rangle$. This article describes some situations where $\alpha$ is injective and some where it is not.

We prove that if $A$ is a right Ore localization of $R[a]$ and $B$ is a right Ore localization of $R[b]$, then $\alpha$ is injective. For example, the group ring over $R$ of the free group on $\{1+a, 1+b\}$ is $R\left[(1+a)^{ \pm 1}\right] \amalg_{R} R\left[(1+b)^{ \pm 1}\right]$, which then embeds in $R\langle\langle a, b\rangle\rangle$. We thus recover a celebrated result of R. H. Fox, via a proof simpler than those previously known.

We show that $\alpha$ is injective if $R$ is $\Pi$-semihereditary, that is, every finitely generated, torsionless, right $R$-module is projective. (This concept was first studied by M.F. Jones, who showed that it is left-right symmetric. It follows from a result of I. I. Sahaev that if w.gl.dim $R \leqslant 1$ and $R$ embeds in a skew field, then $R$ is $\Pi$-semihereditary. Also, it follows from a result of V.C. Cateforis that if $R$ is right semihereditary and right self-injective, then $R$ is $\Pi$-semihereditary.)

The arguments and results extend easily from two variables to any set of variables.

The article concludes with some results contributed by G. M. Bergman that describe situations where $\alpha$ is not injective. He shows that if $R$ is commutative and w.gl.dim $R \geqslant 2$, then there exist examples where the map $\alpha^{\prime}: A \amalg_{R} B \rightarrow R\langle\langle a\rangle\rangle \amalg_{R} R\langle\langle b\rangle\rangle$ is not injective, and hence neither is $\alpha$. It follows from a result of $\mathrm{K}$. R. Goodearl that when $R$ is a commutative, countable, non-self-injective, von Neumann regular ring, the map $\alpha^{\prime \prime}: R\langle\langle a\rangle\rangle \amalg_{R} R\langle\langle b\rangle\rangle \rightarrow R\langle\langle a, b\rangle\rangle$ is not injective. Bergman gives procedures for constructing other examples where $\alpha^{\prime \prime}$ is not injective.
\end{abstract}

2010 Mathematics Subject Classification. Primary: 16S10; Secondary: 20C07, 20 E05. Key words. Ring coproduct, free-group group ring, power series, Ore localization.

\section{Summary of the results}

Some of the terms that we use in this section will be explained in more detail in subsequent sections.

Throughout, we fix a ring $R$ (associative, with 1). By an $R$-ring $S$ we mean a ring $S$ together with a specified ring homomorphism $R \rightarrow S$. We write $\mathrm{U}(R)$ for the multiplicative group of units of $R$. We shall be considering the following.

1.1 Hypotheses. Let $R\langle\langle a, b\rangle\rangle$ denote the power-series $R$-ring in two non-commuting, $R$-centralizing variables, $a$ and $b$. Let $A$ be an $R$-subring of $R\langle\langle a\rangle\rangle$ and $B$ be an $R$-subring of $R\langle\langle b\rangle\rangle$. Let $\alpha$ denote the natural map $A \amalg_{R} B \rightarrow R\langle\langle a, b\rangle\rangle$. 
This article describes some situations where $\alpha$ is injective and some where it is not.

In Section 22 we fix some of the notation, and we use a result of P. M. Cohn to decompose the map from the domain of $\alpha$ to the image of $\alpha$ as a direct sum of $R$-bimodule maps.

In Section 3, in Proposition 3.1, we shall see that if $A$ is a right Ore localization of $R[a]$ and $B$ is a right Ore localization of $R[b]$, then $\alpha$ is injective.

1.2 Example. Let $h:=1+a, H:=\langle h\rangle \leqslant \mathrm{U}(R\langle\langle a\rangle\rangle)$, and let $R H$ denote the group ring of $H$ over $R$. It is not difficult to see that we may view $R[a]$ as $R[h] \subseteq R H \subseteq R\langle\langle a\rangle\rangle$ and that $R H$ is a central Ore localization of $R[h]$. The analogous statements hold for $k:=1+b$ and $K:=\langle k\rangle \leqslant \mathrm{U}(R\langle\langle b\rangle\rangle)$. By Proposition 3.1 below, the map $\alpha: R H \amalg_{R} R K \rightarrow R\langle\langle a, b\rangle\rangle$ is injective. Here, $R H \amalg_{R} R K$ may be identified with the group ring over $R$ of the group $F$ freely generated by two symbols $1+a, 1+b$. We thus recover the celebrated result given in 1953 by R. H. Fox [11, that $R F$ embeds in $R\langle\langle a, b\rangle\rangle$. Although Fox considers only the case $R=\mathbb{Z}$, his argument works for any ring. The proof given here is simpler than the previously known proofs, such as the original proof reviewed recently in [1, pp.366-371], and the proof by G. M. Bergman [8, pp. 528-529] of the case where $R$ is a field.

(One consequence of Fox's result is that if $R \neq\{0\}$, then the group $F$ embeds in $\mathrm{U}(R\langle\langle a, b\rangle\rangle)$. W. Magnus [21] had already given a short proof of the $R=\mathbb{Z}$ case of this consequence in 1935, and deduced much useful information about free groups. Some of this information eventually led to the discovery, in 1946, of the important fact that $F$ is an orderable group. Much later, Bergman [3] remarked that orderability follows directly from Magnus' embedding, since $\mathbb{Z}\langle\langle a, b\rangle\rangle$ can easily be given a ring ordering whose positive cone contains $F$.)

In Section 4, we review some definitions and results from ring theory. We say that $R$ is $\Pi$-semihereditary if every finitely generated, right $R$-submodule of a direct product of copies of $R$ is projective. (Completely reducible rings are clearly $\Pi$-semihereditary, since all their modules are projective. It is also clear that $\Pi$-semihereditary rings are right semihereditary and, hence, satisfy w.gl.dim $R \leqslant 1$.) This concept was studied first by M. F. Jones [16] who showed that it is left-right symmetric. Earlier, K. R. Goodearl [12 had observed that a result of V. C. Cateforis 4 shows that if $R$ is right semihereditary and right self-injective, then $R$ is $\Pi$-semihereditary; see Theorem 4.17 below. It follows from a result of I. I. Sahaev [24] that if w.gl.dim $R \leqslant 1$ and $R$ embeds in a skew field, then $R$ is $\Pi$-semihereditary; see Corollary 4.15(ii) below. We recall these and other examples of П-semihereditary rings.

In Section 5 , we write $\alpha$ as a composite $A \amalg_{R} B \stackrel{\alpha^{\prime}}{\rightarrow} R\langle\langle a\rangle\rangle \amalg_{R} R\langle\langle b\rangle\rangle \stackrel{\alpha^{\prime \prime}}{\rightarrow} R\langle\langle a, b\rangle\rangle$. Then, in Corollary [5.4, we show that $\alpha^{\prime}, \alpha^{\prime \prime}$, and $\alpha$ are injective if $R$ is $\Pi$-semihereditary, or, more generally, if every finitely generated right $R$-submodule of $R\langle\langle a\rangle\rangle$ is projective. The examples of the preceding section then apply.

The results of Sections 3 and 5 extend from two variables to any set of variables; the arguments are very similar but the notation is more complicated. We leave the details to the interested reader.

Section 6 contains results contributed by Bergman that describe situations where $\alpha$ is not injective. Examples of non-injectivity of $\alpha$ can arise from non-injectivity either of $\alpha^{\prime}$ or of $\alpha^{\prime \prime}$. (Examples of the former sort clearly lead to non-injectivity of $\alpha$, while an example of the latter sort does so on taking $A=R\langle\langle a\rangle\rangle, B=R\langle\langle b\rangle\rangle$.) 
Examples are given of both sorts. He obtains non-injectivity of $\alpha^{\prime}$ for any commutative ring $R$ with w.gl.dim $R \geqslant 2$, e.g. $R=\mathbb{Q}[x, y]$. A result of Goodearl [12] yields non-injectivity of $\alpha^{\prime \prime}$ whenever $R$ is a commutative, countable, non-self-injective, von Neumann regular ring, e.g. $R=\mathbb{Q}\left[e_{i}: i \in \mathbb{Z}\right] /\left(e_{i} e_{j}-\delta_{i, j} e_{i}: i, j \in \mathbb{Z}\right)$. Constructions are described that generalize the latter example.

\section{The bimodule structure of ring coproducts}

2.1 Notation. We denote by $\mathbb{N}$ the set of finite cardinals, $\{0,1,2,3, \ldots\}$.

For any $R$-bimodules $M_{1}$ and $M_{2}$, we will denote the $R$-bimodule $M_{1} \otimes_{R} M_{2}$ by $M_{1} \otimes M_{2}$ if $R$ is understood.

For any $R$-bimodule $M$ and any $n \in \mathbb{N}$, we recursively define the $R$-bimodule tensor power $M^{\otimes n}$ by the formulas $M^{\otimes 0}:=R$ and $M^{\otimes(n+1)}:=M^{\otimes n} \otimes M$.

2.2 Notation. Suppose that Hypotheses 1.1 hold.

Let $\{a, b\}^{*}$ denote the free monoid on $\{a, b\}$. We shall usually write elements of $R\langle\langle a, b\rangle\rangle$ as formal sums $\sum f(w) \cdot w$, where $f: w \mapsto f(w)$ is an arbitrary function $w \in\{a, b\}^{*}$

from $\{a, b\}^{*}$ to $R$. The set $\left\{w \in\{a, b\}^{*}: f(w) \neq 0\right\}$ is called the $\{a, b\}^{*}$-support of $\sum f(w) \cdot w$.

$u \in\{a, b\}^{*}$

We view $R \subseteq R[a] \subseteq R\langle\langle a\rangle\rangle \subseteq R\langle\langle a, b\rangle\rangle$ and $R \subseteq R[b] \subseteq R\langle\langle b\rangle\rangle \subseteq R\langle\langle a, b\rangle\rangle$. Let $\mathfrak{a}:=A \cap a R\langle\langle a\rangle\rangle$ and $\mathfrak{b}:=B \cap b R\langle\langle b\rangle\rangle$. Then $\mathfrak{a}$ is a two-sided ideal of $A$, and, as $R$-bimodules, $A=R \oplus \mathfrak{a}$. Analogous statements hold for $B$.

The ring coproduct of $A$ and $B$ amalgamating the two copies of $R$ will be denoted $A \amalg_{R} B$, or by $A \amalg B$ if $R$ is understood. Now $A \amalg B=(R \oplus \mathfrak{a}) \amalg(R \oplus \mathfrak{b})$. As noted by P. M. Cohn [7, pp. 60-61], it is not difficult to show that there is then an expansion as a direct sum of $R$-bimodules

$$
A \amalg B=R \oplus \mathfrak{a} \oplus \mathfrak{b} \oplus(\mathfrak{a} \otimes \mathfrak{b}) \oplus(\mathfrak{b} \otimes \mathfrak{a}) \oplus(\mathfrak{a} \otimes \mathfrak{b} \otimes \mathfrak{a}) \oplus(\mathfrak{b} \otimes \mathfrak{a} \otimes \mathfrak{b}) \oplus \cdots .
$$

We may write this in the form

(1) $A \amalg B=R \oplus \mathfrak{a} \oplus \mathfrak{b} \oplus \bigoplus_{n \geqslant 1}\left((\mathfrak{a} \otimes \mathfrak{b})^{\otimes n} \oplus(\mathfrak{b} \otimes \mathfrak{a})^{\otimes n} \oplus\left((\mathfrak{a} \otimes \mathfrak{b})^{\otimes n} \otimes \mathfrak{a}\right) \oplus\left((\mathfrak{b} \otimes \mathfrak{a})^{\otimes n} \otimes \mathfrak{b}\right)\right)$.

When $\alpha$ is applied to (11), the direct sums are replaced with sums, and the tensor-product multiplications within $A \amalg B$ are replaced with the multiplications of $R\langle\langle a, b\rangle\rangle$. Thus, we may write

$$
\alpha(A \amalg B)=R+\mathfrak{a}+\mathfrak{b}+\sum_{n \geqslant 1}\left((\mathfrak{a} \mathfrak{b})^{n}+(\mathfrak{b} \mathfrak{a})^{n}+\left((\mathfrak{a} \mathfrak{b})^{n} \mathfrak{a}\right)+\left((\mathfrak{b} \mathfrak{a})^{n} \mathfrak{b}\right)\right) .
$$

The $\{a, b\}^{*}$-support of $\mathfrak{a}$ lies in $\left\{a^{i}: i \geqslant 1\right\}$, and the $\{a, b\}^{*}$-support of $\mathfrak{b}$ lies in $\left\{b^{j}: j \geqslant 1\right\}$. It follows that any two summands appearing in (2) have disjoint $\{a, b\}^{*}$-supports. Hence, we recover directness in the summation, and may write

$$
\alpha(A \amalg B)=R \oplus \mathfrak{a} \oplus \mathfrak{b} \oplus \underset{n \geqslant 1}{\bigoplus_{n}}\left((\mathfrak{a} \mathfrak{b})^{n} \oplus(\mathfrak{b} \mathfrak{a})^{n} \oplus\left((\mathfrak{a} \mathfrak{b})^{n} \mathfrak{a}\right) \oplus\left((\mathfrak{b} \mathfrak{a})^{n} \mathfrak{b}\right)\right) .
$$

For any finite alternating string $w$ formed from the letters $a$ and $b$, let us write $\alpha_{w}$ for the map from the corresponding alternating tensor product of factors $\mathfrak{a}$ and $\mathfrak{b}$ in $A \amalg B$ to the corresponding alternating product in $R\langle\langle a, b\rangle\rangle$. Thus, as an $R$-bimodule map, $A \amalg B \rightarrow \alpha(A \amalg B)$ decomposes as the direct sum of the three identity maps $R \stackrel{\alpha_{\emptyset}}{\longrightarrow} R, \mathfrak{a} \stackrel{\alpha_{a}}{\longrightarrow} \mathfrak{a}, \mathfrak{b} \stackrel{\alpha_{b}}{\longrightarrow} \mathfrak{b}$, and the further $R$-bimodule maps, $n \geqslant 1$,

$$
\begin{aligned}
(\mathfrak{a} \otimes \mathfrak{b})^{\otimes n} \stackrel{\alpha_{(a b)^{n}}}{\longrightarrow}(\mathfrak{a} \mathfrak{b})^{n}, & (\mathfrak{b} \otimes \mathfrak{a})^{\otimes n} \stackrel{\alpha_{(b a)^{n}}}{\longrightarrow}(\mathfrak{b} \mathfrak{a})^{n}, \\
(\mathfrak{a} \otimes \mathfrak{b})^{\otimes n} \otimes \mathfrak{a} \stackrel{\alpha_{(a b)^{n} a}}{\longrightarrow}(\mathfrak{a} \mathfrak{b})^{n} \mathfrak{a}, & (\mathfrak{b} \otimes \mathfrak{a})^{\otimes n} \otimes \mathfrak{b} \stackrel{\alpha_{(b a)^{n} b}}{\longrightarrow}(\mathfrak{b} \mathfrak{a})^{n} \mathfrak{b} .
\end{aligned}
$$


Thus $\alpha$ is injective if and only if each of the four maps is injective for all $n \geqslant 1$.

\section{Ore localizations}

We can now give a simple condition that ensures that $\alpha$ is injective.

3.1 Proposition. If Hypotheses 1.1 hold and, moreover, A is a right Ore localization of $R[a]$ and $B$ is a right Ore localization of $R[b]$, then $\alpha$ is injective.

Proof. We use Notation 2.2. We shall show below that for any $n \geqslant 1$, if $\alpha_{(a b)^{n}}$ is injective, then $\alpha_{(a b)^{n} a}$ is injective. The analogous arguments will clearly work for the other three cases required.

Suppose then that $(\mathfrak{a} \otimes \mathfrak{b})^{\otimes n} \stackrel{\alpha_{(a b)^{n}}}{\longrightarrow}(\mathfrak{a} \mathfrak{b})^{n} \subseteq R\langle\langle a, b\rangle\rangle$ is injective. By considering $\{a, b\}^{*}$-supports, we see that, for each $d \geqslant 0,(\mathfrak{a} \otimes \mathfrak{b})^{\otimes n} \otimes R a^{d} \rightarrow(\mathfrak{a} \mathfrak{b})^{n} a^{d}$ is then injective. Again by considering $\{a, b\}^{*}$-supports, we see that the map

$$
\bigoplus_{d \geqslant 0}\left((\mathfrak{a} \otimes \mathfrak{b})^{\otimes n} \otimes\left(R a^{d}\right)\right) \rightarrow \bigoplus_{d \geqslant 0}\left((\mathfrak{a} \mathfrak{b})^{n} a^{d}\right) \subseteq R\langle\langle a, b\rangle\rangle
$$

is injective; that is, the map

$$
(\mathfrak{a} \otimes \mathfrak{b})^{\otimes n} \otimes(R[a]) \rightarrow(\mathfrak{a} \mathfrak{b})^{n}(R[a]) \subseteq R\langle\langle a, b\rangle\rangle
$$

is injective.

Since $A$ is a right Ore localization of $R[a]$, there exists some subset $U$ of $\mathrm{U}(A)$ such that the family $(R[a] u: u \in U)$ forms a directed system under inclusion and the direct limit of the system is $A$. For each $u \in U$, we have a commutative diagram

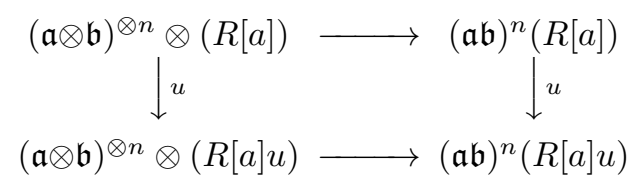

in which the maps on the top and the two sides are bijective. Hence, the map on the bottom,

$$
(\mathfrak{a} \otimes \mathfrak{b})^{\otimes n} \otimes(R[a] u) \rightarrow(\mathfrak{a} \mathfrak{b})^{n}(R[a] u) \subseteq R\langle\langle a, b\rangle\rangle,
$$

is injective. On taking the directed union over $u \in U$, we find that the map

$$
(\mathfrak{a} \otimes \mathfrak{b})^{\otimes n} \otimes A \rightarrow(\mathfrak{a} \mathfrak{b})^{n} A \subseteq R\langle\langle a, b\rangle\rangle
$$

is injective. By considering $\{a, b\}^{*}$-supports, we see that the latter map is the direct sum of the two maps $\alpha_{(a b)^{n}}$ and $\alpha_{(a b)^{n} a}$. Hence, $\alpha_{(a b)^{n} a}$ is injective.

The other three inductive implications can be proved in the same way. The injectivity of $\alpha$ then follows by induction.

3.2 Remarks. In Example 1.2, we noted that one case of Proposition 3.1 is the result of Fox [11] that the natural map $R F \rightarrow R\langle\langle a, b\rangle\rangle$ is injective, where $F$ is the free group on two symbols $1+a, 1+b$. Fox's proof introduced $R$-linear endomorphisms of $R F$ that are now called Fox derivatives; see [8, Example 7, p. 55]. To indicate one important connection between the above proof of Proposition 3.1 and Fox's original argument, we remark that right multiplication by $a$ gives a bijective map

$$
(\mathfrak{a} \otimes \mathfrak{b})^{\otimes n} \otimes\left(R a^{d}\right) \rightarrow(\mathfrak{a} \otimes \mathfrak{b})^{\otimes n} \otimes\left(R a^{d+1}\right),
$$

and the inverse map has the same action as that of the left Fox derivative on $R F$ with respect to $1+a$ relative to the free generating set $\{1+a, 1+b\}$.

The original motivation of this article was to show that the proof of the embedding became more transparent when more emphasis was placed on the ring-theoretic viewpoint. 


\section{Review of П-semihereditary rings}

In this section, we review some of the history of what we call $\Pi$-semihereditary rings. This will provide us with some examples to which the results of the next section apply. We shall recall the proofs since they are all fairly short, scattered over the literature, and not always available in the formulation that will be required. No new results are claimed, but we have tried to give proofs that minimize background requirements.

4.1 Definitions. For each of the definitions given below, the dual left-right definition will also be understood.

Let $M$ be a left $R$-module.

(1) Let $X$ and $Y$ be sets.

For $m, n \in \mathbb{N}$, we let ${ }^{m} Y^{n}$ denote the set of $m \times n$ matrices with coordinates in $Y$. Absence of an exponent is to be read as an exponent 1.

Let $\prod_{X} Y$ denote the set whose elements are the functions $X \rightarrow Y, x \mapsto y_{x}$, represented as families $\left(y_{x}: x \in X\right)$. We shall sometimes write $\prod_{X} Y$ as $Y^{X}$, and think of the elements intuitively as ' $1 \times X$ matrices'. Similarly, we sometimes write $\prod_{X} Y$ as ${ }^{X} Y$, and think of the elements intuitively as ' $X \times 1$ matrices'.

In a natural way, $\prod_{X} R$ is an $R$-bimodule and $\prod_{X} M$ is a left $R$-module. By the multiplication map $\left(\prod_{X} R\right) \otimes M \rightarrow\left(\prod_{X} R\right) M \subseteq \prod_{X}^{X} M$, we shall mean the left $R$-module map determined by $\left(r_{x}: x \in X\right) \otimes m \mapsto\left(r_{x} m: x \in X\right)$. We shall sometimes let $\left({ }^{X} R\right) \otimes M \rightarrow\left({ }^{X} R\right) M \subseteq{ }^{X} M$ denote the underlying additive map; here, ${ }^{X} R$ has a right $R$-module structure and ${ }^{X} M$ has an abelian group structure. When $M$ is an $R$-bimodule, the right $R$-action is to be maintained in all cases.

When $M=\prod_{Y} R$, we let $\mu_{X, Y}$ denote the foregoing multiplication map. Here, we have an $R$-bimodule map

$$
\begin{gathered}
\left(\prod_{X} R\right) \otimes\left(\prod_{Y} R\right) \stackrel{\mu_{X, Y}}{\longrightarrow} \prod_{X \times Y} R, \\
\left(r_{x}: x \in X\right) \otimes\left(s_{y}: y \in Y\right) \mapsto\left(r_{x} s_{y}:(x, y) \in X \times Y\right) .
\end{gathered}
$$

We shall sometimes denote the underlying additive map by ${ }^{X} R \otimes R^{Y} \stackrel{\mu_{X, Y}}{\longrightarrow}{ }^{X} R^{Y}$, where we think of the elements of ${ }^{X} R^{Y}$ imprecisely as ' $X \times Y$ matrices'.

(2) We say that $M$ is torsionless if there exists an injective left $R$-module map from $M$ to a direct product of copies of $R$. Clearly, submodules of torsionless modules are torsionless.

(3) One says that $M$ is coherent if every finitely generated submodule is finitely presented. Clearly, submodules of coherent modules are coherent.

The ring $R$ is left coherent if $R$ is coherent as left $R$-module, that is, every finitely generated left ideal of $R$ is finitely presented as left $R$-module.

The ring $R$ is left $\Pi$-coherent if, for every set $Y, R^{Y}$ is coherent as left $R$-module, or, equivalently, every finitely generated, torsionless, left $R$-module is finitely presented. This property was studied first by Jones [16, p. 103]. Clearly, left П-coherent rings are left coherent.

(4) We say that $M$ is semihereditary if every finitely generated submodule is projective. Clearly, semihereditary modules are coherent, and submodules of semihereditary modules are semihereditary.

The ring $R$ is left semihereditary if $R$ is semihereditary as left $R$-module, that is, every finitely generated left ideal of $R$ is projective as left $R$-module. 
We now come to the property of interest to us, which was studied first by Jones [16], who showed that it is left-right symmetric; see Theorem 4.11 below. We say that the ring $R$ is $\Pi$-semihereditary if, for every set $X,{ }^{X} R$ is semihereditary as right $R$-module, or, equivalently, every finitely generated, torsionless right $R$-module is projective. Prior to Jones' work, Goodearl [12] had mentioned that right self-injective, von Neumann regular rings have this property; see Theorem 4.17 below.

Clearly, П-semihereditary rings are right semihereditary and right $\Pi$-coherent.

(5) We say that $M$ is flat if, for all injective right $R$-module maps $K \rightarrow F$, the induced map $K \otimes_{R} M \rightarrow F \otimes_{R} M$ is injective, or, equivalently, for all finitely generated right ideals $I$ of $R$, the multiplication map $I \otimes_{R} M \rightarrow I M \subseteq M=R \otimes_{R} M$ is injective. See [23, Theorem 3.53]. Notice that it is therefore sufficent to consider inclusion maps $K \rightarrow F$ where $F$ is a finitely generated, free, right $R$-module and $K$ is a finitely generated $R$-submodule of $F$.

If every finitely generated submodule of $M$ is flat, then $M$ is flat. More generally, if $M$ is the direct limit of a directed system of flat modules, then $M$ is flat. See [23, Theorem 3.47].

If $M$ is flat, then every left $R$-summand of $M$ is flat. See [23, Theorem 3.45].

We write w.gl.dim $R \leqslant 1$ if every finitely generated left ideal of $R$ is flat as left $R$-module, or, equivalently, every submodule of every flat left $R$-module is flat, or equivalently every submodule of every flat right $R$-module is flat; see 23 , Theorem 9.24]. Otherwise, we write w.gl.dim $R \geqslant 2$.

(6) For any infinite cardinal $\kappa$, we say that $R$ is left $\kappa$-Noetherian if every left ideal of $R$ is generated by $\kappa$ (or fewer) elements. For example, if $|R| \leqslant \aleph_{0}$, then $R$ is left $\aleph_{0}$-Noetherian. Also, if $|R| \geqslant \aleph_{0}$, then $R$ is left $|R|$-Noetherian.

The next result is a variant of [6, Theorem 2.4] suitable for our purposes.

4.2 Theorem. (Cohn, 1959) For any flat left $R$-module $F$ and left $R$-submodule $K$ of $F$, the following are equivalent.

(a) $F / K$ is left $R$-flat.

(b) For all $\ell, m \in \mathbb{N}$ and $y \in{ }^{\ell} R^{m},\left(y\left({ }^{m} F\right)\right) \cap\left({ }^{\ell} K\right) \subseteq y\left({ }^{m} K\right)$ in ${ }^{\ell} F$.

Proof. If $\ell, m \in \mathbb{N}$ and $y \in{ }^{\ell} R^{m}$, then ${ }^{\ell} R$ may be considered an arbitrary finitely generated, free, right $R$-module and $y\left({ }^{m} R\right)$ an arbitrary finitely generated, right $R$-submodule of ${ }^{\ell} R$. Thus, the left $R$-module $M:=F / K$ is flat if and only if the natural map

$$
\left(y\left({ }^{m} R\right)\right) \otimes_{R} M \rightarrow\left({ }^{\ell} R\right) \otimes_{R} M
$$

is injective for all $\ell, m, y$.

Suppose that $\ell, m$, and $y$ are given.

We have an exact sequence

$$
\left(y\left({ }^{m} R\right)\right) \otimes_{R} K \rightarrow\left(y\left({ }^{m} R\right)\right) \otimes_{R} F \rightarrow\left(y\left({ }^{m} R\right)\right) \otimes_{R} M \rightarrow 0,
$$

and we shall re-interpret each term. Since $F$ is left $R$-flat, $\left(y\left({ }^{m} R\right)\right) \otimes_{R} F$ embeds in $\left({ }^{\ell} R\right) \otimes_{R} F={ }^{\ell} F$, and the image is $y\left({ }^{m} F\right)$. Hence, we may make the identification $\left(y\left({ }^{m} R\right)\right) \otimes_{R} F=y\left({ }^{m} F\right)$. The image of $\left(y\left({ }^{m} R\right)\right) \otimes_{R} K$ in $\left(y\left({ }^{m} R\right)\right) \otimes_{R} F=y\left({ }^{m} F\right)$ is then $y\left({ }^{m} K\right)$. Using (44), we now find that we may make the identification $\left(y\left({ }^{m} R\right)\right) \otimes_{R} M=\left(y\left({ }^{m} F\right)\right) /\left(y\left({ }^{m} K\right)\right)$.

In the special case where $y$ is an identity matrix, we have the (obvious) identification $\left({ }^{\ell} R\right) \otimes_{R} M=\left({ }^{\ell} F\right) /\left({ }^{\ell} K\right)$. We now find that we may identify (3) with the natural map $\left(y\left({ }^{m} F\right)\right) /\left(y\left({ }^{m} K\right)\right) \rightarrow\left({ }^{\ell} F\right) /\left({ }^{\ell} K\right)$. Then (国) $\Leftrightarrow($ b) follows.

The next two results are taken from [5, Proposition 2.2 and Corollary], attributed to O. E. Villamayor. 
4.3 Theorem. (Villamayor, 1960) For any $n \in \mathbb{N}$ and left $R$-submodule $K$ of $R^{n}$, the following are equivalent.

(a) $R^{n} / K$ is left $R$-flat.

(b) For all $\ell \in \mathbb{N}$ and $x \in{ }^{\ell} K \subseteq{ }^{\ell} R^{n}, x \in x\left({ }^{n} K\right)$.

Proof. The case $F=R^{n}$ of Theorem 4.2 allows us to rewrite (ai) as

(a) For all $\ell, m \in \mathbb{N}$ and $y \in{ }^{\ell} R^{m},\left(y\left({ }^{m} R^{n}\right)\right) \cap\left({ }^{\ell} K\right) \subseteq y\left({ }^{m} K\right)$ in ${ }^{\ell} R^{n}$.

$($ a $) \Rightarrow($ b $)$. Suppose $x \in{ }^{\ell} K$. Taking $m=n$ and $y=x$ in (a) we obtain $\left(x\left({ }^{n} R^{n}\right)\right) \cap\left({ }^{\ell} K\right) \subseteq x\left({ }^{n} K\right)$ in ${ }^{\ell} R^{n}$. Then $x=x \mathbf{I}_{n} \in x\left({ }^{n} R^{n}\right) \cap\left({ }^{\ell} K\right) \subseteq x\left({ }^{n} K\right)$.

(b) $\Rightarrow($ a $)$. Suppose $y \in{ }^{\ell} R^{m}$. Consider any $x \in\left(y\left({ }^{m} R^{n}\right)\right) \cap\left({ }^{\ell} K\right)$. We have $x \in{ }^{\ell} K$, and then, by (b),$x \in(x)\left({ }^{n} K\right) \subseteq\left(y\left({ }^{m} R^{n}\right)\right)\left({ }^{n} K\right) \subseteq y\left({ }^{m} K\right)$.

4.4 Theorem. (Villamayor, 1960) For any left R-module $M$, the following are equivalent.

(a) $M$ is finitely generated and projective.

(b) $M$ is finitely presented and flat.

Proof. (a) $\Rightarrow(\mathrm{b})$ is clear.

$($ b $) \Rightarrow($ (a) $)$. Since $M$ is a finitely presented left $R$-module, there exist $n \in \mathbb{N}$ and a finitely generated left $R$-submodule $K$ of $R^{n}$ such that $M=R^{n} / K$. Since $K$ is finitely generated, there exist $\ell \in \mathbb{N}$ and $x \in{ }^{\ell} K \subseteq{ }^{\ell} R^{n}$ such that $K=\left(R^{\ell}\right) x$. By Theorem 4.3 (国) $\Rightarrow(\mathrm{b})$, since $M$ is $R$-flat, $x \in x\left({ }^{n} K\right)$, that is, there exists some $e \in{ }^{n} K$ such that $x=x \cdot e$. Now the left $R$-module map $R^{n} \rightarrow K, y \mapsto y \cdot e$, acts as the identity on $K$ because it does so on the rows of $x$, which form a generating set of $K$. This proves that $R^{n} / K$ is projective.

The following result is a variant of [20, Satz 2] which is the same as [9, Proposition 2.1], attributed to M. Auslander.

4.5 Theorem. (Lenzing, 1969 \& Auslander, 1970) For any finitely generated left $R$-module $M$, the following are equivalent.

(a) $M$ is finitely presented.

(b) For every set $X$, the multiplication map $\left({ }^{X} R\right) \otimes_{R} M \rightarrow{ }^{X} M$ is injective.

(c) For some infinite set $X$ such that $R$ is left $|X|$-Noetherian, the multiplication map $\left({ }^{X} R\right) \otimes_{R} M \rightarrow{ }^{X} M$ is injective.

Proof. ((a) $\Rightarrow$ (b). By (a), there exist $m, n \in \mathbb{N}$ and an exact sequence of left $R$-modules $R^{m} \rightarrow R^{n} \rightarrow M \rightarrow 0$. We then have a commutative diagram with exact rows:

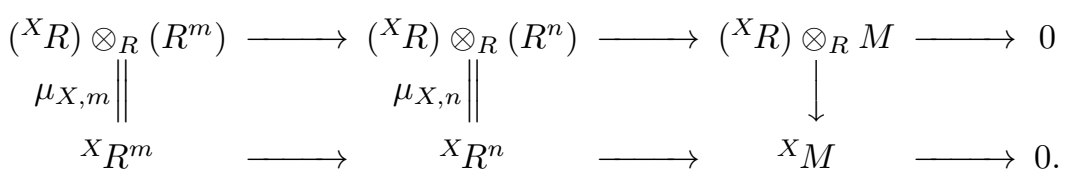

Hence, $\left({ }^{X} R\right) \otimes_{R} M \rightarrow{ }^{X} M$ is injective (and surjective).

(b) $\Rightarrow$ (c) is clear.

$($ c $) \Rightarrow$ (a) . By hypothesis, there exist $n \in \mathbb{N}$ and an exact sequence of left $R$-modules $0 \rightarrow K \rightarrow R^{n} \rightarrow M \rightarrow 0$, and it suffices to show that $K$ is finitely generated. We then have a commutative diagram with exact rows:

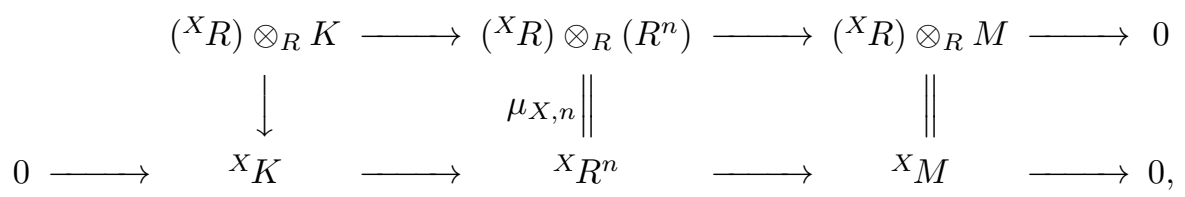


where $\left({ }^{X} R\right) \otimes_{R} M \rightarrow{ }^{X} M$ is injective by (IC), and is here easily seen to be surjective. Hence, $\left({ }^{X} R\right) \otimes_{R} K \rightarrow{ }^{X} K$ is surjective, that is, $\left({ }^{X} R\right) K={ }^{X} K$. Since $R$ is left $|X|$-Noetherian by (ㄷ) $K$ is $|X|$-generated; hence, there exists some generating family $\left(k_{x}: x \in X\right)$ which may be viewed as an element of ${ }^{X} K$, and it then has the form $\sum_{i=1}^{m}\left(r_{x, i}: x \in X\right) k_{i}^{\prime}$, since ${ }^{X} K=\left({ }^{X} R\right) K$. Then, for each $x \in X, k_{x}=\sum_{i=1}^{m} r_{x, i} k_{i}^{\prime}$. Hence, $K=\sum_{x \in X} R k_{x} \subseteq \sum_{i=1}^{m} R k_{i}^{\prime} \subseteq K$. Thus, $K$ is finitely generated.

The next two results are taken from [5, Theorems 2.1 and 4.1].

4.6 Theorem. (Chase, 1960) The following are equivalent.

(a) $R$ is left coherent, i.e. every finitely generated left ideal is finitely presented.

(b) For every finitely generated left ideal $I$ of $R$ and every set $X$, the multiplication map $\left({ }^{X} R\right) \otimes_{R} I \rightarrow\left({ }^{X} R\right) I \subseteq{ }^{X} I \subseteq{ }^{X} R=\left({ }^{X} R\right) \otimes_{R} R$ is injective.

(c) For every set $X,{ }^{X} R$ is right $R$-flat.

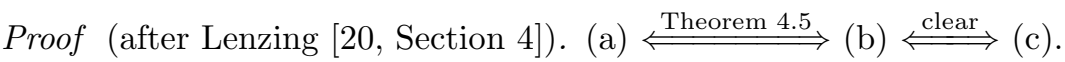

4.7 Theorem. (Chase, 1960) The following are equivalent.

(a) $R$ is left semihereditary, i.e. every finitely generated left ideal is projective.

(b) w.gl.dim $R \leqslant 1$ and $R$ is left coherent.

(c) w.gl.dim $R \leqslant 1$ and, for every set $X,{ }^{X} R$ is right $R$-flat.

(d) Every torsionless right $R$-module is flat.

(e) Every finitely generated, torsionless, right $R$-module is flat.

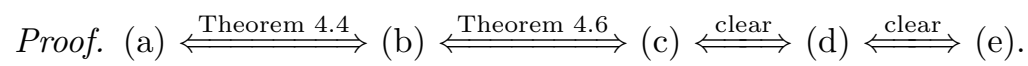

Goodearl [12, Theorem 1] generalized Theorem4.5 to the non-finitely generated case by showing that, for any left $R$-module $M$, all of the multiplication maps $\left({ }^{X} R\right) \otimes_{R} M \rightarrow{ }^{X} M$ are injective if and only if, for each finitely generated submodule $N$ of $M$, the inclusion map $N \rightarrow M$ factors through a finitely presented module. We record the special case we shall be using and its special proof. Recall the meaning of $\mu_{X, Y}$ from Definitions 4.1(1).

4.8 Theorem. (Goodearl, 1972) For any non-empty set $Y$, the following are equivalent.

(a) $R^{Y}$ is coherent as left $R$-module.

(b) $R$ is a left coherent ring, and, for every set $X, \mu_{X, Y}$ is injective.

(c) $R$ is a left coherent ring, and, for some infinite set $X$ such that $R$ is left $|X|$-Noetherian, $\mu_{X, Y}$ is injective.

Proof. Let $M:=R^{Y}$, and let $\mathcal{M}$ denote the directed family of finitely generated left $R$-submodules of $M$.

$($ (a) $\Rightarrow(\mathrm{b})$. Since $Y$ is non-empty, $R$ embeds in $M$, and, hence, $R$ is coherent as left $R$-module, that is, $R$ is a left coherent ring. Let $N \in \mathcal{M}$. By (a),$N$ is finitely presented. By Theorem 4.5 (a) $\Rightarrow($ b $)$, the multiplication map $\left({ }^{X} R\right) \otimes N \rightarrow{ }^{X} N \subseteq{ }^{X} M$ is injective. On taking the direct limit of the system of injective maps $\left({ }^{X} R\right) \otimes N \rightarrow{ }^{X} M$ over all $N \in \mathcal{M}$, we see that $\left({ }^{X} R\right) \otimes M \stackrel{\mu_{X, Y}}{\longrightarrow}{ }^{X} M$ is injective, as desired.

(b) $\Rightarrow$ (c) is clear.

$($ (c) $) \Rightarrow($ a $)$ (after Herbera-Trlifaj [13, Corollary 2.11] ). Let $N \in \mathcal{M}$ and consider the natural commutative diagram 


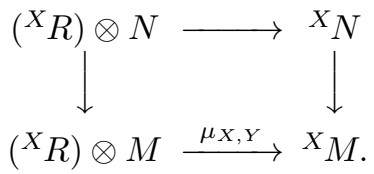

By Theorem 4.6 (国) $\Rightarrow$ (Ic),${ }^{X} R$ is right $R$-flat, and, hence, the left map is injective. By (ㄷ), the bottom map is injective. Hence, the top map is injective. By Theorem 4.5 $($ (c) $\Rightarrow($ (a) $), N$ is finitely presented. Thus, $M$ is coherent as left $R$-module.

The following result is a variant of [2, (4.5)] that is suitable for our purposes.

4.9 Theorem. (Bass, 1960) Let $Y$ be an infinite set such that $R$ is right $|Y|$-Noetherian. If $M$ is a finitely generated, torsionless, left $R$-module, then $M$ embeds in $R^{Y}$. Hence, $R$ is a left $\Pi$-coherent ring if and only if $R^{Y}$ is a coherent left $R$-module.

Proof. Since $M$ is a finitely generated left $R$-module, on dualizing we find that the right $R$-module $M^{*}:=\operatorname{Hom}_{R}\left(M,{ }_{R} R\right)$ embeds in a finitely generated, free, right $R$-module. Since $R$ is right $|Y|$-Noetherian, $M^{*}$ is $|Y|$-generated. On dualizing we find that the left $R$-module $M^{* *}:=\operatorname{Hom}_{R}\left(M^{*}, R_{R}\right)$ embeds in $R^{Y}$. Since $M$ is torsionless, the double-dual map $M \rightarrow M^{* *}$ is injective, and $M$ embeds in $R^{Y}$.

4.10 Corollary. The following are equivalent.

(a) $R$ is a left $\Pi$-coherent ring, i.e. every finitely generated, torsionless, left module is finitely presented.

(b) $R$ is a left coherent ring and, for all sets $X$ and $Y, \mu_{X, Y}$ is injective.

(c) $R$ is a left coherent ring and, for some infinite sets $X$ and $Y$ such that $R$ is left $|X|$-Noetherian and right $|Y|$-Noetherian, $\mu_{X, Y}$ is injective.

Proof. This follows from Theorems 4.8 and 4.9

We now recall the aspect of [16, Theorem 2.11] that is of interest to us.

4.11 Theorem. (Jones, 1982) The following are equivalent.

(a) $R$ is left and right semihereditary, and, for all sets $X, Y, \mu_{X, Y}$ is injective.

(b) $R$ is left and right coherent, w.gl.dim $R \leqslant 1$, and, for all sets $X, Y, \mu_{X, Y}$ is injective.

(c) $R$ is left coherent, w.gl. $\operatorname{dim} R \leqslant 1$, and $R$ is right $\Pi$-coherent.

(d) Every finitely generated, torsionless, right $R$-module is flat and finitely presented.

(e) $R$ is П-semihereditary, i.e. every finitely generated, torsionless, right $R$-module is projective.

These conditions are also equivalent to their left-right duals.

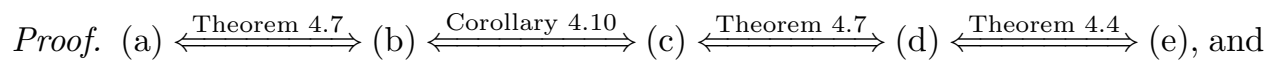
(a) is left-right symmetric.

In the remainder of this section, we recall some types of rings that are $\Pi$-semihereditary. We first present a result from [24].

4.12 Theorem. (Sahaev, 1965) For each $n \in \mathbb{N}$ and each $y \in{ }^{n} R^{n}$, let $\dot{y}$ denote $\mathbf{I}_{n}-y$. The following are equivalent.

(a) There exists a finitely generated, non-finitely presented, flat, left $R$-module $M$.

(b) There exist $n \in \mathbb{N}$ and a function $\mathbb{N} \rightarrow{ }^{n} R^{n}, k \mapsto y_{k}$, such that, for each $k \in \mathbb{N}, y_{k} \dot{y}_{k+1}=\mathbf{0}_{n} \neq y_{k+1} \dot{y}_{k} ;$ equivalently, $y_{k} y_{k+1}=y_{k}$ and $y_{k+1} y_{k} \neq y_{k+1}$; equivalently, $\dot{y}_{k} \dot{y}_{k+1}=\dot{y}_{k+1}$, and $\dot{y}_{k+1} \dot{y}_{k} \neq \dot{y}_{k}$. 
Proof. $(\underline{\mathrm{a}}) \Rightarrow(\mathrm{b})$. Since $M$ is finitely generated and not finitely presented, there exist $n \in \mathbb{N}$ and a non-finitely generated left $R$-submodule $K$ of $R^{n}$ such that $M=R^{n} / K$. We view $R^{n}$ as an $\left(R,{ }^{n} R^{n}\right)$-bimodule.

We shall recursively construct a sequence $\left(y_{k}: k \in \mathbb{N}\right)$ in ${ }^{n} K \subseteq{ }^{n} R^{n}$. Set $y_{0}:=\mathbf{0}_{n} \in{ }^{n} K$. We may now suppose that $k \in \mathbb{N}$ and $y_{k} \in{ }^{n} K$. Since $K$ is not finitely generated, it is easy to see that there exists some $x_{k} \in{ }^{n+1} K \subseteq{ }^{n+1} R^{n}$ such that $\left(R^{n}\right) y_{k} \subset\left(R^{n+1}\right) x_{k} \subseteq K$. By Theorem 4.3 (国) $\Rightarrow($ b $)$, since $M$ is flat, $x_{k} \in x_{k}\left({ }^{n} K\right)$, that is, there exists some $y_{k+1} \in{ }^{n} K$ such that $x_{k}=x_{k} y_{k+1}$. Hence, $x_{k} \dot{y}_{k+1}=\mathbf{0}_{n}$. Now, $\left(R^{n}\right) y_{k} \dot{y}_{k+1} \subseteq\left(R^{n+1}\right) x_{k} \dot{y}_{k+1}=\{0\}$, and we see that $y_{k} \dot{y}_{k+1}=\mathbf{0}_{n}$.

Also, $\left(R^{n}\right) y_{k+1} y_{k} \subseteq\left(R^{n}\right) y_{k} \subset\left(R^{n+1}\right) x_{k}=\left(R^{n+1}\right) x_{k} y_{k+1} \subseteq\left(R^{n}\right) y_{k+1}$, and we see that $y_{k+1} y_{k} \neq y_{k+1}$. Thus, $y_{k+1} \dot{y}_{k} \neq \mathbf{0}_{n}$.

This completes the recursive construction of the sequence, and (b) holds.

$($ b $) \Rightarrow$ (a) . Let $F$ denote the $\left(R,{ }^{n} R^{n}\right)$-bimodule $R^{n}$. Then $\left(F y_{k}: k \in \mathbb{N}\right)$ is a family of left $R$-submodules of $F$. For each $k \in \mathbb{N}, F y_{k}=F y_{k} y_{k+1} \subseteq F y_{k+1}$; also $F y_{k+2} \dot{y}_{k+1} \neq\{0\}=F y_{k} \dot{y}_{k+1}$, and, hence, $F y_{k+2} \neq F y_{k}$. Thus, $\left(F y_{k}: k \in \mathbb{N}\right)$ is a non-stationary, ascending chain of left $R$-submodules of $F$.

Set $K:=\bigcup_{k=0}^{\infty}\left(F y_{k}\right)$ and $M:=F / K$. Then $M$ is a finitely generated, non-finitely presented, left $R$-module. To prove (国), it suffices to show that $M$ is left $R$-flat, and here we may apply Theorem $4.3(\underline{\mathrm{b}}) \Rightarrow($ a) $)$. Let $\ell \in \mathbb{N}$ and $x \in{ }^{\ell} K$; it remains to show that $x \in x\left({ }^{n} K\right)$. As $x \in{ }^{\ell} K=\bigcup_{k=0}^{\infty}\left({ }^{\ell}\left(F y_{k}\right)\right)$, there exists some $k \in \mathbb{N}$ such that $x \in{ }^{\ell}\left(F y_{k}\right)$. Then $x \dot{y}_{k+1}=0$. Since each row of $y_{k+1}$ lies in $K$, $y_{k+1} \in{ }^{n} K$. Now, $x=x y_{k+1} \in x\left({ }^{n} K\right)$, as desired.

4.13 Definitions. (1) We shall say that $R$ is a left Sahaev ring if every finitely generated, flat, left $R$-module is finitely presented, or, equivalently, projective, by Theorem 4.4. The term 'left S-ring' is sometimes used in the literature.

Every subring of a left Sahaev ring is again a left Sahaev ring, since these are the rings for which the condition in Theorem 4.12(b) fails. In particular, rings that embed in skew fields are left and right Sahaev rings.

It is clear from the foregoing definition that left Noetherian rings are left Sahaev rings. Both Sahaev [24] and S. Jøndrup [15, Lemma 1.4] discuss connections between the condition in Theorem 4.12(b) and various chain conditions.

(2) A commutative Prüfer domain is a semihereditary, commutative domain. A commutative Bézout domain is a commutative domain in which every finitely generated ideal is principal.

(3) One says that $R$ is a semifir if every finitely generated left ideal of $R$ is free of unique rank, as left $R$-module, and similarly on the right. It is sufficient to assume the condition on the left; see [8, Theorem I.1.1].

We now present [15, Corollary 2].

4.14 Theorem. (Jøndrup, 1971) The following hold.

(i) If $R$ is right semihereditary and not left Sahaev, then $R$ is not right Sahaev.

(ii) If w.gl.dim $R \leqslant 1$ and $R$ is right Sahaev, then $R$ is left Sahaev and $\Pi$-semihereditary.

Proof. (ii) We apply Theorem $4.12($ (a) $) \Rightarrow($ b $)$, and we change $y_{0}$ to $\mathbf{0}_{n}$. We shall speak of the kernel and image of each element of ${ }^{n} R^{n}$ viewed as an $R$-endomorphism of ${ }^{n} R$ acting on the left by matrix multiplication.

Let $k \in \mathbb{N}$. Since $y_{k+2} \dot{y}_{k+1} \neq \mathbf{0}_{n}=y_{k} \dot{y}_{k+1}, \operatorname{Ker}\left(y_{k+2}\right) \nsupseteq \operatorname{Im}\left(\dot{y}_{k+1}\right) \subseteq \operatorname{Ker}\left(y_{k}\right)$. Hence, $\operatorname{Ker}\left(y_{k+2}\right) \neq \operatorname{Ker}\left(y_{k}\right)$. Clearly $\dot{y}_{k+1}$ acts as the identity on $\operatorname{Ker}\left(y_{k+1}\right)$, and we see that $\operatorname{Ker}\left(y_{k+1}\right) \subseteq \operatorname{Im}\left(\dot{y}_{k+1}\right) \subseteq \operatorname{Ker}\left(y_{k}\right)$. Hence, $\operatorname{Ker}\left(y_{2 k+2}\right) \subset \operatorname{Ker}\left(y_{2 k}\right)$. Since $R$ is right semihereditary, $\operatorname{Im}\left(y_{2 k+2}\right)$ is a projective $R$-submodule of ${ }^{n} R$, and, 
hence, $\operatorname{Ker}\left(y_{2 k+2}\right)$ is an $R$-summand of ${ }^{n} R$, and, therefore, of $\operatorname{Ker}\left(y_{2 k}\right)$. Thus, $\operatorname{Ker}\left(y_{2 k}\right)=\operatorname{Ker}\left(y_{2 k+2}\right) \oplus L_{k}$ for some $L_{k} \neq\{0\}$. Then,

$$
{ }^{n} R=\operatorname{Ker}\left(y_{0}\right)=\operatorname{Ker}\left(y_{2 k+2}\right) \oplus\left(\bigoplus_{i=0}^{k} L_{i}\right) .
$$

Each term of the directed system $\left({ }^{n} R /\left(\bigoplus_{i=0}^{k} L_{i}\right): k \in \mathbb{N}\right)$ is then $R$-projective. On taking the direct limit, we see that ${ }^{n} R /\left(\bigoplus_{i=0}^{\infty} L_{i}\right)$ is finitely generated, not finitely presented, and flat. Hence, $R$ is not right Sahaev.

(ii) As w.gl.dim $R \leqslant 1$, every finitely generated right ideal of $R$ is flat, and hence projective, since $R$ is right Sahaev. Thus, $R$ is right semihereditary. By the contrapositive of (i), $R$ is left Sahaev. Hence, $R$ is left semihereditary. By Theorem 4.7 $(\underline{\text { a }}) \Rightarrow($ 远 $)$, every finitely generated, torsionless, right $R$-module is flat, and hence projective, since $R$ is right Sahaev.

4.15 Corollary. The following hold.

(i) If w.gl.dim $R \leqslant 1$ (e.g. $R$ is left or right (semi)hereditary) and $R$ is left or right Sahaev (e.g. $R$ is left or right Noetherian or $R$ embeds in a skew field), then $R$ is $\Pi$-semihereditary.

(ii) If $R$ is a commutative principal ideal domain, or more generally, a commutative Bézout domain, or, even more generally, a commutative Prüfer domain, then $R$ is $\Pi$-semihereditary.

Proof. (ii) is Theorem 4.14(ii) and its left-right dual, while (iii) gives some cases of (ii) where $R$ is semihereditary and embeds in a field.

The following is [14, Theorem 2B], attributed to Cohn.

4.16 Theorem. (Cohn, 1969) Semifirs are left and right semihereditary, left and right Sahaev rings. Hence, semifirs are П-semihereditary.

Proof (after Jøndrup [15, Corollary 3]). It is clear that semifirs are left and right semihereditary. From the proof of Theorem 4.14(1), it may be seen that if $R$ is right semihereditary and not left Sahaev, then, for some $n \in \mathbb{N},{ }^{n} R$ is a direct sum of $n+1$ non-zero right $R$-submodules; this cannot happen if $R$ is a semifir. Hence semifirs are left (and right) Sahaev rings, and Corollary 4.15(ii) applies.

We now turn to the final topic of this section, self-injective rings; for background on injective modules, see [23, pp. 65-68].

At the beginning of the proof of [12, Theorem $2(\mathrm{c}) \Rightarrow(\mathrm{a})]$, Goodearl notes the consequence of [4, Theorem $2.1(\mathrm{a}) \Rightarrow(\mathrm{b})]$ that, over a right self-injective, von Neumann regular ring, every torsionless right module is projective. This seems to have been one of the very earliest occasions on which the projectivity of finitely generated torsionless modules was considered.

4.17 Theorem. (Cateforis, 1969) If $R$ is right semihereditary and right self-injective, then $R$ is $\Pi$-semihereditary.

Proof. By definition, $R$ is $\Pi$-semihereditary if each finitely generated, torsionless, right $R$-module $M$ is projective. Since $M$ is finitely generated, we may write $M=F / K$ where $F$ is a finitely generated, free, right $R$-module, and $K$ is a submodule of $F$. In particular, $F$ is right $R$-injective. Let $\mathcal{L}$ denote the set of submodules of $F$ whose intersection with $K$ is $\{0\}$. Clearly, $\mathcal{L} \neq \emptyset$. By Zorn's lemma, $\mathcal{L}$ contains some $\subseteq$-maximal element $L$. Then $F \supseteq K \oplus L$. We then have injective right $R$-module maps $K \rightarrow F / L, k \mapsto k+L$, and $K \rightarrow F, k \mapsto k$. Since $F$ is right $R$-injective, the domain of the latter map can be transported along the former map to give a right $R$-module map $\phi: F / L \rightarrow F$ such that, for all $k \in K, \phi(k+L)=k$. 
Define $\phi^{\prime}: F \rightarrow F$ by $f \mapsto \phi(f+L)$. Then $\operatorname{Ker}\left(\phi^{\prime}\right) \supseteq L$, and, for all $k \in K$, $\phi^{\prime}(k)=k$. If $\operatorname{Im}\left(\phi^{\prime}\right)=K$, then $F / K$ is projective, as desired. Thus, it suffices to assume that $\operatorname{Im}\left(\phi^{\prime}\right) \supset K$, and obtain a contradiction.

Define $\phi^{\prime \prime}: F \rightarrow M$ by $f \mapsto \phi^{\prime}(f)+K \in F / K=M$. Then there exists some $m \in \operatorname{Im}\left(\phi^{\prime \prime}\right)-\{0\}$. Thus, $F \supset \operatorname{Ker}\left(\phi^{\prime \prime}\right) \supseteq K \oplus L$.

Since $M$ is torsionless, we may compose $\phi^{\prime \prime}$ with a map from $M$ to $R$ which does not vanish on $m$, and thus obtain a map $\psi: F \rightarrow R$ with $F \supset \operatorname{Ker}(\psi) \supseteq K \oplus L$. Then $\operatorname{Im}(\psi)$ is a finitely generated right ideal of $R$. Since $R$ is right semihereditary, $\operatorname{Im}(\psi)$ is projective. Hence, $F=\operatorname{Ker}(\psi) \oplus L^{\prime}$ for some $L^{\prime} \neq\{0\}$. Now $F \supseteq K \oplus L \oplus L^{\prime}$ and this contradicts the $\subseteq$-maximality of $L$ in $\mathcal{L}$, as desired.

4.18 Remarks. Recall that if $R$ is von Neumann regular, then every finitely presented right $R$-module is projective by Theorem 4.4 hence every finitely generated right ideal of $R$ is a summand of $R$, and hence $R$ is right semihereditary.

Recall also that if $R$ is right semihereditary and right self-injective, then every finitely generated right ideal is projective, hence injective, hence a summand of $R$, and $R$ is von Neumann regular.

We shall be using [22, Corollary], which is as follows.

4.19 Theorem. (Osofsky, 1964) If $R$ is right self-injective and right hereditary, then $R$ is completely reducible.

Proof. Suppose not. We shall obtain a contradiction. Let $\mathcal{U}$ denote the set of right ideals of $R$ that are not finitely generated. By Remarks 4.18, $R$ is von Neumann regular; since $R$ is not completely reducible, $\mathcal{U} \neq \emptyset$. By Zorn's lemma, $\mathcal{U}$ contains some $\subseteq$-maximal element $I$.

Since $R$ is right hereditary, $I$ is right $R$-projective. Since $R$ is von Neumann regular, results of Kaplansky show that $I$ is a direct sum of infinitely many nonzero right ideals of $R$; in the case where $I$ is $\aleph_{0}$-generated, this follows from [18, Proof of Lemma 1], and in the case where $I$ is not $\aleph_{0}$-generated, it follows from [17, Theorem 1]. There then exists some decomposition $I=I^{\prime} \oplus I^{\prime \prime}$ with $I^{\prime}, I^{\prime \prime} \in \mathcal{U}$.

We shall now show that there exists some right ideal $\overline{I^{\prime}}$ of $R$ with the properties that $I^{\prime} \subseteq \overline{I^{\prime}}, \overline{I^{\prime}} \notin \mathcal{U}$, and $\overline{I^{\prime}} \cap I^{\prime \prime}=\{0\}$; in Remarks 4.20 , we shall give an alternative argument which does not require background on injective hulls. Recall that Eckmann and Schopf [10] showed that, since $R$ is right self-injective, $I^{\prime}$ has a right $R$-injective hull $\overline{I^{\prime}}$ in $R$ which is a maximal right $R$-essential extension of $I^{\prime}$ in $R$; see [23, proof of Theorem $3.30(\mathrm{iii}) \Rightarrow(\mathrm{i})]$. Then $\overline{I^{\prime}}$ is a right $R$-summand of $R$, and, hence, $\overline{I^{\prime}}$ is right $R$-cyclic. Thus, $\overline{I^{\prime}} \notin \mathcal{U}$. Also, since $I^{\prime} \cap I^{\prime \prime}=\{0\}$, it follows that $\overline{I^{\prime}} \cap I^{\prime \prime}=\{0\}$, as claimed.

Since $\overline{I^{\prime}} \notin \mathcal{U}$, we have $I \neq \overline{I^{\prime}}$ and $I \subset \overline{I^{\prime}}$. Since $I^{\prime \prime} \in \mathcal{U}$, it can be seen that $\overline{I^{\prime}} \oplus I^{\prime \prime} \in \mathcal{U}$. Now $I=I^{\prime} \oplus I^{\prime \prime} \subset \overline{I^{\prime}} \oplus I^{\prime \prime}$. This contradicts the maximality of $I$ in $\mathcal{U}$ and completes the proof.

4.20 Remarks. Here is an alternative proof of the existence of an $\overline{I^{\prime}}$. Let $M:=R / I^{\prime}$, and consider the double-dual map $\phi: M \rightarrow M^{* *}$ as in the proof of Theorem 4.9. There exists a right ideal $\overline{I^{\prime}}$ of $R$ such that $\operatorname{Ker}(\phi)=\overline{I^{\prime}} / I^{\prime}$, and then $\operatorname{Im}(\phi) \simeq R / \overline{I^{\prime}}$. By Theorem 4.17, $R$ is right $\Pi$-coherent. It is clear that $\operatorname{Im}(\phi)$ is a finitely generated, torsionless, right $R$-module, and, hence, $\operatorname{Im}(\phi)$ is finitely presented. Thus, $\overline{I^{\prime}}$ is finitely generated, and, hence, $\overline{I^{\prime}} \notin \mathcal{U}$. We shall now show that $\overline{I^{\prime}} \cap I^{\prime \prime}=\{0\}$. Consider any $x \in I^{\prime \prime}$. Then $x R=e R$ for some idempotent $e \in R$, since $R$ is von Neumann regular. Since $e R \subseteq I^{\prime \prime}$, we see that $e R \cap I^{\prime}=\{0\}$, and, hence, $e R$ embeds in $R / I^{\prime}=M$. Since $R$ is right self-injective, $e R$ is right $R$-injective. Thus, $e R$ becomes a summand of $M$ which, as $e R$ is projective, 
must then embed in $M^{* *}$. We now see that $e R \cap \overline{I^{\prime}}=\{0\}$. It then follows that $\overline{I^{\prime}} \cap I^{\prime \prime}=\{0\}$, as claimed.

The following combines [12, Theorem $2(\mathrm{a}) \Rightarrow(\mathrm{c})]$ and the commutative case of Theorem 4.19

4.21 Theorem. Let $R$ be a commutative, $\Pi$-coherent, von Neumann regular ring.

(i) (Goodearl, 1972) $R$ is self-injective.

(ii) (Osofsky, 1964) If $R$ is $\aleph_{0}$-Noetherian or, more generally, hereditary, then $R$ is completely reducible.

Proof. (1i) (based loosely on Kobayashi [19, Theorem 2]) By Baer's criterion [23, Theorem 3.20], to show that $R$ is self-injective, it suffices to show that, for each ideal $I$ of $R$, each $R$-module map $\phi: I \rightarrow R$ is given by multiplication by some element of $R$.

Now $\pi_{1}: R^{2} \rightarrow R,(x, y) \mapsto x$, and $\psi: R^{2} \rightarrow R^{I},(x, y) \mapsto(\phi(x \cdot i)-y \cdot i: i \in I)$, are $R$-module maps. Then $\operatorname{Im}(\psi)$ is a finitely generated, torsionless $R$-module. Since $R$ is $\Pi$-coherent, $\operatorname{Ker}(\psi)$ is a finitely generated $R$-submodule of $R^{2}$. Hence, $\pi_{1}(\operatorname{Ker}(\psi))$ is a finitely generated ideal of $R$. Since $R$ is von Neumann regular, there exists some idempotent $e \in R$ such that $\pi_{1}(\operatorname{Ker}(\psi))=R \cdot e$. For each $x \in R$, we now see that $x \in R \cdot e$ if and only if there exists some $y \in R$ such that, for each $i \in I$, $\phi(x \cdot i)=y \cdot i$, that is, $\phi(i \cdot x)=i \cdot y$, since $R$ is commutative.

We apply the 'if' part taking $x \in I$ and $y=\phi(x)$. For each $i \in I$, we have $\phi(i \cdot x)=i \cdot \phi(x)=i \cdot y$. Hence, $x \in R \cdot e$. Thus, $I \subseteq R \cdot e$ and $i \cdot e=i$ for all $i \in I$.

We now apply the 'only if' part taking $x=e$. There exists some $y \in R$ such that, for each $i \in I, \phi(i \cdot e)=i \cdot y$, that is, $\phi(i)=i \cdot y$, as desired.

(ii) Recall that a right $\aleph_{0}$-Noetherian, von Neumann regular ring is right hereditary, by [18, Lemma 1]. Now (iii) follows from (ii) and Theorem 4.19.

4.22 Remarks. If w.gl. $\operatorname{dim} R \geqslant 2$, then $R$ is not $\Pi$-semihereditary.

If w.gl.dim $R=1$ and $R$ is right Sahaev, then $R$ is left Sahaev and $\Pi$-semihereditary, by Theorem 4.14(ii). This class includes all the semifirs that are not skew fields, by Theorem 4.16. It also includes all the Prüfer domains that are not fields.

If w.gl.dim $R=0$ and $R$ is left or right self-injective, then $R$ is $\Pi$-semihereditary, by Theorem 4.17. This class includes all the right semihereditary, right self-injective rings. It also includes all the commutative $\Pi$-semihereditary rings which have w.gl.dim $R=0$, by Theorem 4.21(i). It also includes all the completely reducible rings.

If w.gl.dim $R=0$ and $R$ is right Sahaev, then clearly every finitely generated right $R$-module is projective, and $R$ is then completely reducible. Such rings are included in the previous case.

Thus, we have two disjoint classes of examples of П-semihereditary rings.

\section{If $R$ is $\Pi$-semihereditary, then $\alpha$ is injective}

For the remainder of the article, we fix the following.

5.1 Notation. For each $n \in \mathbb{N}$, we have a multiplication map that will be denoted $\left(\prod_{\mathbb{N}} R\right)^{\otimes n} \stackrel{\beta_{n}}{\longrightarrow} \prod_{\mathbb{N}^{n}} R$.

Whenever Hypotheses 1.1 hold, we have a natural factorization of $\alpha$ that will be denoted $A \amalg_{R} B \stackrel{\alpha^{\prime}}{\rightarrow} R\langle\langle a\rangle\rangle \amalg_{R} R\langle\langle b\rangle\rangle \stackrel{\alpha^{\prime \prime}}{\longrightarrow} R\langle\langle a, b\rangle\rangle$. 
5.2 Proposition. If Hypotheses 1.1 hold and $A, B$, and $\prod_{\mathbb{N}} R$ are left and right $R$-flat, then $A \amalg_{R} B \stackrel{\alpha^{\prime}}{\longrightarrow} R\langle\langle a\rangle\rangle \amalg_{R} R\langle\langle b\rangle\rangle$ is injective.

Proof. Clearly, the $R$-bimodule map $\prod_{\mathbb{N}} R \rightarrow a R\langle\langle a\rangle\rangle,\left(r_{i}: i \in \mathbb{N}\right) \mapsto \sum_{i \geqslant 0}\left(r_{i} a^{i+1}\right)$, is bijective. Similarly for $b R\langle\langle b\rangle\rangle$.

We see that $a R\langle\langle a\rangle\rangle$ and $b R\langle\langle b\rangle\rangle$ are left and right $R$-flat.

We use Notation 2.2. Now, $A=R \oplus \mathfrak{a}$ and $B=R \oplus \mathfrak{b}$; hence, $\mathfrak{a}$ and $\mathfrak{b}$ are left and right $R$-flat.

Let $n \in \mathbb{N}$. For $1 \leqslant i \leqslant n$, let $M_{i}$ denote one of $a R\langle\langle a\rangle\rangle$, a, $b R\langle\langle b\rangle\rangle$, or $\mathfrak{b}$. It follows by induction that the $R$-bimodule $M_{1} \otimes M_{2} \otimes \cdots \otimes M_{n}$ is left and right $R$-flat. It then follows by induction that each of the natural maps

$$
\begin{aligned}
(\mathfrak{a} \otimes \mathfrak{b})^{\otimes n} & \rightarrow(a R\langle\langle a\rangle\rangle \otimes b R\langle\langle b\rangle\rangle)^{\otimes n}, \\
(\mathfrak{b} \otimes \mathfrak{a})^{\otimes n} & \rightarrow(b R\langle\langle b\rangle\rangle \otimes a R\langle\langle a\rangle\rangle)^{\otimes n}, \\
(\mathfrak{a} \otimes \mathfrak{b})^{\otimes n} \otimes \mathfrak{a} & \rightarrow(a R\langle\langle a\rangle\rangle \otimes b R\langle\langle b\rangle\rangle)^{\otimes n} \otimes a R\langle\langle a\rangle\rangle, \\
(\mathfrak{b} \otimes \mathfrak{a})^{\otimes n} \otimes \mathfrak{b} & \rightarrow(b R\langle\langle b\rangle\rangle \otimes a R\langle\langle a\rangle\rangle)^{\otimes n} \otimes b R\langle\langle b\rangle\rangle,
\end{aligned}
$$

is injective. By using the standard $R$-bimodule decompositions of $A \amalg B$ and $R\langle\langle a\rangle\rangle \amalg R\langle\langle b\rangle\rangle$, we see that, as an $R$-bimodule map, $\alpha^{\prime}$ is a direct sum of these injective maps, and hence is injective.

5.3 Proposition. Suppose that Hypotheses 1.1 hold.

(i) $R\langle\langle a\rangle\rangle \amalg_{R} R\langle\langle b\rangle\rangle \stackrel{\alpha^{\prime \prime}}{\longrightarrow} R\langle\langle a, b\rangle\rangle$ is injective if and only if, for each $n \in \mathbb{N}$, $\left(\prod_{\mathbb{N}} R\right)^{\otimes n} \stackrel{\beta_{n}}{\longrightarrow} \prod_{\mathbb{N}^{n}} R$ is injective.

(ii) If $R$ is left or right coherent and $\beta_{2}$ is injective, then $\alpha^{\prime \prime}$ is injective.

(iii) If $\prod_{\mathbb{N}} R$ is left or right $R$-coherent, then $\alpha^{\prime \prime}$ is injective.

Proof. (i) We may assume that $A=R\langle\langle a\rangle\rangle, B=R\langle\langle b\rangle\rangle$ and $\alpha=\alpha^{\prime \prime}$. We use Notation 2.2. Let $n \in \mathbb{N}$. Consider the map $(\mathfrak{a} \otimes \mathfrak{b})^{\otimes n} \rightarrow(\mathfrak{a} \mathfrak{b})^{n} \subseteq R\langle\langle a, b\rangle\rangle$. The $R$-bimodule isomorphisms $\prod_{\mathbb{N}} R \rightarrow \mathfrak{a}$ and $\prod_{\mathbb{N}} R \rightarrow \mathfrak{b}$ give an embedding $\left(\prod_{\mathbb{N}} R\right)^{\otimes(2 n)} \rightarrow R\langle\langle a\rangle\rangle \amalg R\langle\langle b\rangle\rangle$ with image $(\mathfrak{a} \otimes \mathfrak{b})^{\otimes n}$. They also give an embed$\operatorname{ding} \prod_{\mathbb{N}^{2 n}} R \rightarrow R\langle\langle a, b\rangle\rangle$ with image $\widehat{(\mathfrak{a b})^{n}}$, the closure of $(\mathfrak{a} \mathfrak{b})^{n}$ in $R\langle\langle a, b\rangle\rangle$. Thus the map $(\mathfrak{a} \otimes \mathfrak{b})^{\otimes n} \rightarrow \widehat{(\mathfrak{a} \mathfrak{b})^{n}}$ is a copy of $\beta_{2 n}$. We now see that $\alpha$ is a direct sum of $R$-bimodule maps that consist of one copy of $\beta_{0}$ and two copies of $\beta_{n}$ for each $n \geqslant 1$. In particular, $\alpha$ is injective if and only if all the $\beta_{n}, n \in \mathbb{N}$, are injective.

(ii) By symmetry, we may assume that $R$ is left coherent.

We shall show by induction that, for each $n \in \mathbb{N}, \beta_{n}$ is injective. It is clear that $\beta_{0}$ is injective. Suppose that $n \geqslant 0$, and that $\beta_{n}$ is injective. Notice that $\beta_{n+1}$ may be factored as

$$
\left(\prod_{\mathbb{N}} R\right) \otimes\left(\left(\prod_{\mathbb{N}} R\right)^{\otimes n}\right) \stackrel{\left(\prod_{\mathbb{N}} R\right) \otimes \beta_{n}}{\longrightarrow}\left(\prod_{\mathbb{N}} R\right) \otimes\left(\prod_{\mathbb{N}^{n}} R\right) \stackrel{\beta_{2}^{\prime}}{\longrightarrow} \prod_{\mathbb{N}^{n+1}} R .
$$

Since $R$ is left coherent, by Theorem 4.6 (国) $\Rightarrow(\mathbb{C}), \prod_{\mathbb{N}} R$ is right $R$-flat, and, hence, $\left(\prod_{\mathbb{N}} R\right) \otimes \beta_{n}$ is injective. Since there exists a bijection between $\mathbb{N}^{n}$ and $\mathbb{N}$, we see that $\beta_{2}^{\prime}$ may be identified with $\beta_{2}$ and is then injective. Hence, the composition $\beta_{n+1}$ is injective. This completes the inductive argument. By (i), $\alpha^{\prime \prime}$ is injective.

(iii) By symmetry, we may assume that $\prod_{\mathbb{N}} R$ is left $R$-coherent. By Theo$\operatorname{rem} 4.8(\mathrm{a}) \Rightarrow(\mathrm{b}), R$ is left coherent and $\beta_{2}$ is injective. By (ii), $\alpha^{\prime \prime}$ is injective. 
5.4 Corollary. With Hypotheses 1.1, the following hold.

(i) If $\prod_{\mathbb{N}} R$ is a left or right semihereditary $R$-module, then $\alpha$ is injective.

(ii) If $R$ is a $\Pi$-semihereditary ring, then $\alpha$ is injective.

(iii) If $R$ is right semihereditary (e.g. w.gl.dim $R=0)$ and $R$ is right self-injective, then $\alpha$ is injective (and w.gl.dim $R=0$ ).

(iv) If w.gl.dim $R \leqslant 1$ (e.g. $R$ is left or right semihereditary) and $R$ is left or right Sahaev, then $\alpha$ is injective (and $R$ is left and right semihereditary and left and right Sahaev).

Proof. (i) By symmetry, we may assume that $\prod_{\mathbb{N}} R$ is left $R$-semihereditary. By Proposition 5.3 (iii), $\alpha^{\prime \prime}$ is injective.

Notice that $\prod_{\mathbb{N}} R$ is left $R$-coherent and that every left $R$-submodule of $\prod_{\mathbb{N}} R$ is

flat. In particular, $R$ is left coherent and w. gl. $\operatorname{dim} R \leqslant 1$. By Theorem 4.6 (国) $\Rightarrow($ (c) $)$, $\prod_{\mathbb{N}} R$ is right $R$-flat, and then all its right $R$-submodules are flat. Hence, $\prod_{\mathbb{N}} R, R\langle\langle a\rangle\rangle$, $R\langle\langle b\rangle\rangle, A$, and $B$, are left and right $R$-flat. By Proposition $5.2, \alpha^{\prime}$ is injective.

Hence, $\alpha$ is injective.

(ii) follows from (i).

(iii) follows from (ii) and Theorem 4.17

(iv) follows from (ii) and Corollary 4.15(ii).

In the next section, we shall see results of Bergman and Goodearl that show that $\alpha$ can fail to be injective whenever $R$ is a commutative ring such that either w. gl. $\operatorname{dim} R \geqslant 2$ or $R$ is $\aleph_{0}$-Noetherian, non-self-injective, and von Neumann regular.

\section{Non-injectivity phenomena}

In this section, we give examples contributed by G. M. Bergman where Hypotheses 1.1 hold and $\alpha$ is not injective. Throughout, we use the factorization of $\alpha$ as $A \amalg_{R} B \stackrel{\alpha^{\prime}}{\rightarrow} R\langle\langle a\rangle\rangle \amalg_{R} R\langle\langle b\rangle\rangle \stackrel{\alpha^{\prime \prime}}{\longrightarrow} R\langle\langle a, b\rangle\rangle$.

We begin with a general example where $R[a] \subseteq A \subseteq R\left[a,(1+a)^{-1}\right]$ and $R[b] \subseteq B \subseteq R\left[b,(1+b)^{-1}\right]$, but $\alpha^{\prime}$ is not injective.

6.1 Proposition. (Bergman) Suppose that $R$ has two-sided ideals $I, J$ such that the multiplication map $I \otimes J \rightarrow I J$ is not injective, e.g. $R=\mathbb{Z} / p^{2} \mathbb{Z}, p$ prime, or $R=\mathbb{Q}[x, y]$. Then, with a suitable choice of $A$ and $B$, Hypotheses 1.1 hold and $\alpha^{\prime}$, $\alpha$ are not injective.

Proof. For $R=\mathbb{Z} / p^{2} \mathbb{Z}, p$ prime, we may take $I=J=p R$, where $p \otimes p \mapsto 0$.

For $R=\mathbb{Q}[x, y]$, we may take $I=J=x R+y R$, where $(x \otimes y)-(y \otimes x) \mapsto 0$.

Let $h:=1+a$ and $H:=\langle h\rangle \leqslant \mathrm{U}(R\langle\langle a\rangle\rangle)$. Then $R[h]=R[a]$, and we view $R[h] \subseteq R H \subseteq R\langle\langle a\rangle\rangle$. We have $R$-bimodule decompositions

$$
R H=\bigoplus_{i \in \mathbb{Z}} h^{i} R=R[h] \oplus \bigoplus_{i \leqslant-1}\left(h^{i} R\right)
$$

In $R H$, take $A$ to be the subring $R[h] \oplus \bigoplus_{i \leqslant-1}\left(h^{i} I\right)$. Since $h^{-1}=1-h^{-1} a, R+a A=A$.

Let $k:=1+b, K:=\langle k\rangle \leqslant \mathrm{U}(R\langle\langle b\rangle\rangle)$. We view $R[b]=R[k] \subseteq R K \subseteq R\langle\langle b\rangle\rangle$, and we have an $R$-bimodule decomposition

$$
R K=R[k] \oplus \underset{i \leqslant-1}{\bigoplus}\left(R k^{i}\right)
$$


In $R K$, take $B$ to be the subring $R[k] \oplus \bigoplus_{i \leqslant-1}\left(J k^{i}\right)$. Since $k^{-1}=1-k^{-1} b, R+b B=B$. We use Notation 2.2, Here,

$$
\mathfrak{a}=a A=a R[h] \oplus \bigoplus_{i \leqslant-1}\left(h^{i} a I\right), \quad \text { and } \quad \mathfrak{b}=b B=b R[k] \oplus \underset{i \leqslant-1}{\bigoplus}\left(J b k^{i}\right) .
$$

In particular, $h^{-1} a I$ is an $R$-bimodule direct summand of $\mathfrak{a}$, and $J b k^{-1}$ is an $R$-bimodule direct summand of $\mathfrak{b}$. Hence $\left(h^{-1} a I\right) \otimes\left(J b k^{-1}\right)$ may be viewed as an $R$-bimodule direct summand of $\mathfrak{a} \otimes \mathfrak{b}$, and also of $A \amalg B$. This summand does not map injectively to $\left(h^{-1} a I\right)\left(J b k^{-1}\right)$ in $(R H) \amalg(R K)$. It follows that $\alpha^{\prime}$ is not injective in this case. Hence, $\alpha$ is not injective.

Recall that w.gl.dim $R \geqslant 2$ if and only if there exists a right ideal $I$ of $R$ that is not right $R$-flat; recall also that $I$ is not right $R$-flat if and only if there exists a left ideal $J$ of $R$ such that the multiplication map $I \otimes_{R} J \rightarrow I J$ is not injective. The next result then follows from Proposition 6.1

6.2 Corollary. Suppose that $R$ is commutative and that w.gl.dim $R \geqslant 2$. Then with a suitable choice of $A$ and $B$, Hypotheses 1.1 hold and $\alpha$ is not injective.

6.3 Remarks. Suppose that $R$ is a commutative Sahaev ring and that Hypotheses 1.1 hold.

By Corollary 6.2, if $\alpha$ is injective for all choices of $A$ and $B$, then w.gl.dim $R \leqslant 1$.

Conversely, by Corollaries 5.4 and 4.15 (ii), if w.gl.dim $R \leqslant 1$, then $\alpha$ is injective.

Thus, among the commutative Sahaev rings $R$, the ones for which $\alpha$ is always injective are the ones with w.gl.dim $R \leqslant 1$, that is, the semihereditary ones.

Hence, among the commutative domains $R$, the ones for which $\alpha$ is always injective are the commutative Prüfer domains.

Also, among the commutative Noetherian rings $R$, the ones for which $\alpha$ is always injective are the hereditary ones.

We now give examples where Hypotheses 1.1 hold and $\beta_{2}$ is not injective, and, hence, by Proposition 5.3(i), $\alpha^{\prime \prime}$ is not injective. These are then examples where $A=R\langle\langle a\rangle\rangle$ and $B=R\langle\langle b\rangle\rangle$ in Hypotheses 1.1 and $\alpha$ is not injective. We remark that if we had been working in the setting of commutative rings of power series, then $\alpha^{\prime \prime}$ would have looked like $\beta_{2}$.

The following result gives a method for constructing examples where $\beta_{2}$ is not injective.

6.4 Proposition. (Bergman) Let $Q$ be a field. For $i=1,2$, let $R_{i}$ be a commutative, augmented $Q$-ring whose augmentation ideal $I_{i}$ is not finitely generated, and let $X_{i}$ be an infinite set such that $I_{i}$ is $\left|X_{i}\right|$-generated. Let $R:=\left(R_{1} \otimes_{Q} R_{2}\right) /\left(I_{1} \otimes_{Q} I_{2}\right)$, a commutative $Q$-ring. Then $\mu_{X_{1}, X_{2}}:\left({ }^{X_{1}} R\right) \otimes_{R}\left(R^{X_{2}}\right) \rightarrow{ }^{X_{1}} R^{X_{2}}$ is not injective.

In detail, for $i=1,2$, any family $m_{i}:=\left(r_{i, j_{i}}: j_{i} \in X_{i}\right)$ that generates $I_{i}$ as ideal of $R_{i}$ may be viewed as an element of $\prod_{X} R$, and then $m_{1} \otimes m_{2}$ is a non-zero element of $\operatorname{Ker}\left(\mu_{X_{1}, X_{2}}\right)$.

Proof. Notice that $R_{1} \otimes_{Q} R_{2}=\left(Q \oplus I_{1}\right) \otimes_{Q}\left(Q \oplus I_{2}\right)=Q \oplus I_{1} \oplus I_{2} \oplus\left(I_{1} \otimes_{Q} I_{2}\right)$, $R=Q \oplus I_{1} \oplus I_{2}, R_{1}$ and $R_{2}$ are subrings of $R, I_{1}$ and $I_{2}$ are ideals of $R$ with product zero, and $R$ is a commutative augmented $Q$-ring with augmentation ideal $I:=I_{1} \oplus I_{2}$.

Here, $\mu_{X_{1}, X_{2}}$ carries $m_{1} \otimes m_{2}$ to $\left(r_{1, j_{1}} r_{2, j_{2}}:\left(j_{1}, j_{2}\right) \in X_{1} \times X_{2}\right)$, which is zero, by our definition of $R$. Thus, it remains to show that $m_{1} \otimes m_{2} \neq 0$ in $\left({ }^{X_{1}} R\right) \otimes_{R}\left(R^{X_{2}}\right)$, and we shall do this by finding a quotient $R$-module in which the image of $m_{1} \otimes m_{2}$ is not zero. 
Let $M_{1}:={ }^{X_{1}}\left(R / I_{2}\right)$, a quotient $R$-module of ${ }^{X_{1}} R$ with trivial $I_{2}$-action. Let $M_{2}:=\left(R / I_{1}\right)^{X_{2}}$, a quotient $R$-module of $R^{X_{2}}$ with trivial $I_{1}$-action. For $i=1,2$, set $\bar{M}_{i}:=M_{i} /\left(M_{i} I\right)=M_{i} /\left(M_{i} I_{i}\right)$, a quotient $R$-module of $\prod_{X_{i}} R$ with trivial $I$-action, and let $\bar{m}_{i}$ denote the image of $m_{i}$ in $\bar{M}_{i}$. Then we have a map

$$
\left({ }^{X_{1}} R\right) \otimes_{R}\left(R^{X_{2}}\right) \rightarrow \bar{M}_{1} \otimes_{R} \bar{M}_{2}=\bar{M}_{1} \otimes_{Q} \bar{M}_{2},
$$

with $m_{1} \otimes_{R} m_{2}$ mapping to $\bar{m}_{1} \otimes_{Q} \bar{m}_{2}$. It suffices to show that $\bar{m}_{1} \otimes_{Q} \bar{m}_{2} \neq 0$, and, since $Q$ is a field, it suffices to show that $\bar{m}_{1} \neq 0$ and $\bar{m}_{2} \neq 0$. By symmetry it suffices to assume that $\bar{m}_{1}=0$ and obtain a contradiction, as follows.

The natural bijection $R_{1} \rightarrow R / I_{2}$ induces a bijection ${ }^{X_{1}} R_{1} \rightarrow M_{1}$, and it carries $\left({ }^{X_{1}} R_{1}\right) I_{1}$ to $M_{1} I_{1}$. Since $m_{1} \in{ }^{X_{1}} R_{1}$ and $\bar{m}_{1}=0$, we have $m_{1} \in\left({ }^{X_{1}} R_{1}\right) I_{1}$. Hence, there is some expression $m_{1}=\sum_{k=1}^{\ell} v_{k} r_{k}$ with each $v_{k} \in{ }^{X_{1}} R_{1}$ and each $r_{k} \in I_{1}$. For each $j_{1} \in X_{1}$, the $j_{1}$ st coordinate of $m_{1}$ is $r_{1, j_{1}}$, and, hence, $r_{1, j_{1}}$ lies in the ideal of $R_{1}$ generated by $\left\{r_{k}: 1 \leqslant k \leqslant \ell\right\}$, a finite subset of $I_{1}$. It follows that $I_{1}$ is a finitely generated ideal of $R_{1}$. This is a contradiction, as desired.

The following expands on the last two paragraphs of [12].

6.5 Proposition. If $R$ is a commutative, $\aleph_{0}$-Noetherian, von Neumann regular ring that is not completely reducible, then the map $\alpha^{\prime \prime}: R\langle\langle a\rangle\rangle \amalg R\langle\langle b\rangle\rangle \rightarrow R\langle\langle a, b\rangle\rangle$ is not injective.

Proof. By Theorem 4.21(ii), $R$ is not $\Pi$-coherent. By the contrapositive of Corollary $4.10(\mathbb{C}) \Rightarrow\left(\right.$ 国), $\beta_{2}$ is not injective. By Proposition 5.3(i), $\alpha^{\prime \prime}$ is not injective.

6.6 Example. If $Q$ is a field and $R=Q\left[e_{j}: j \in \mathbb{N}\right] /\left(e_{k} e_{\ell}-\delta_{k, \ell} e_{k}: k, \ell \in \mathbb{N}\right)$, then $\alpha^{\prime \prime}$ is not injective, since $R$ satisfies the hypotheses of Proposition 6.5 the countable case was first seen in the last two paragraphs of [12]. Let us give an alternative proof by showing that this $R$ has the form considered in Proposition 6.4 with $X_{1}$ and $X_{2}$ countable. Partition $\mathbb{N}$ into two infinite subsets $X_{1}$ and $X_{2}$. For $i=1,2$, let $m_{i}:=\left\{e_{j}: j \in X_{i}\right\}$, let $I_{i}$ denote the ideal of $R$ generated by $m_{i}$, and let $R_{i}$ denote the $Q$-subring of $R$ generated by $m_{i}$. Then we may make the identification $R=\left(R_{1} \otimes_{Q} R_{2}\right) /\left(I_{1} \otimes_{Q} I_{2}\right)$, and, by Proposition 6.4, $m_{1} \otimes m_{2}$ represents a non-zero element of $\operatorname{Ker}\left(\mu_{X_{1}, X_{2}}\right)$. We may identify $\mu_{X_{1}, X_{2}}$ with $\beta_{2}$.

Here is a result similar to Proposition 6.4, which uses a different, rather curious argument to prove that an appropriate element of $\operatorname{Ker} \mu_{X, X}$ is not zero.

6.7 Proposition. (Bergman) Let $Q$ be a non-zero commutative ring, $X$ an infinite set, $I$ the free $Q$-module on $X, R$ the commutative augmented $Q$-ring $Q \oplus I$ with $I^{2}=\{0\}$, and $m$ the element $(x: x \in X)$ of $\prod_{X} R$. Then $m \otimes m$ represents a non-zero element of $\operatorname{Ker}\left(\mu_{X, X}\right)$.

Proof. It is clear that $m \otimes_{R} m$ is an element of $\operatorname{Ker}\left(\mu_{X, X}\right)$, and it remains to show that $m \otimes_{R} m \neq 0$ in $\left({ }^{X} R\right) \otimes_{R}\left(R^{X}\right)$; we shall do this by finding a quotient $R$-module in which the image of $m \otimes_{R} m$ is not zero.

Define $R$-bimodules $P:={ }^{X}\left(R \otimes_{Q} R\right)^{X}$ and $\bar{P}:=(R / I) \otimes_{R} P \otimes_{R}(R / I)$, and make the identification $\bar{P}=P /(I P+P I)$. We have natural $R$-bimodule maps

$$
\left({ }^{X} R\right) \otimes_{Q}\left(R^{X}\right) \rightarrow{ }^{X}\left(R \otimes_{Q} R\right)^{X}=P \rightarrow \bar{P} .
$$

Since $I$ acts trivially on $\bar{P}$ on the left and the right, the $R$-bimodule map $\left({ }^{X} R\right) \otimes_{Q}\left(R^{X}\right) \rightarrow \bar{P}$ is $R$-centralizing, and, hence, factors through the universal 
$R$-centralizing quotient $\left({ }^{X} R\right) \otimes_{R}\left(R^{X}\right)$ of the domain. Under the resulting map $\left({ }^{X} R\right) \otimes_{R}\left(R^{X}\right) \rightarrow \bar{P}$, our element $m \otimes_{R} m$ is mapped to $p+I P+P I$ where $p:=\left(x \otimes_{Q} y:(x, y) \in X \times X\right) \in P$. Thus to show that $m \otimes_{R} m \neq 0$, it suffices to show that $p \notin I P+P I$.

For every $x, y \in X$ and $t \in R \otimes_{Q} R$, we shall write $c_{x \otimes y}(t) \in Q$ for the coefficient of $x \otimes y$ in the expression for $t$ with respect to the $Q$-basis $(X \cup\{1\}) \otimes(X \cup\{1\})$ of $R \otimes_{Q} R$.

Consider any $q=\left(q_{x, y}:(x, y) \in X \times X\right) \in P$. For each $x_{0} \in X$, it can be seen that $x_{0} P={ }^{X}\left(x_{0} Q \otimes_{Q} R\right)^{X}$. Hence, if $q \in x_{0} P$, then, for all $(x, y) \in X \times X$, $c_{x \otimes y}\left(q_{x, y}\right)=0$ if $x \neq x_{0}$. Similar statements hold for $P x_{0}$. If $q \in I P+P I$, then there exist finite subsets $X_{0}, Y_{0} \subseteq X$ such that $q \in \sum_{x_{0} \in X_{0}} x_{0} P+\sum_{y_{0} \in Y_{0}} P y_{0}$. Since $X$ is infinite, there exist $x \in X-X_{0}$ and $y \in X-Y_{0}$, and then $c_{x \otimes y}\left(q_{x, y}\right)=0$. Since $Q \neq\{0\}, c_{x \otimes y}\left(q_{x, y}\right) \neq 1$. Hence, $q \neq p$. This shows that $m \otimes_{R} m \neq 0$, as desired.

In the previous two propositions, we have created elements of $\operatorname{Ker}\left(\mu_{X_{1}, X_{2}}\right)$ of the form $m_{1} \otimes m_{2}$, and this requires the existence of zero-divisors in $R$. By using elements of $\operatorname{Ker}\left(\mu_{X_{1}, X_{2}}\right)$ of the form $m_{1} \otimes m_{2}-m_{3} \otimes m_{4}$, we can avoid having zero-divisors in $R$, as follows.

6.8 Proposition. (Bergman) Let $Q$ be a field, and let $R$ be the subring of $Q[x, y]$ generated by $\left\{x^{i} y: i \in \mathbb{N}\right\}$. In particular, $R$ is a commutative domain. In $\prod_{\mathbb{N}} R$, let

$$
m_{1}:=m_{2}:=\left(x^{2 i+1} y: i \in \mathbb{N}\right), \quad m_{3}:=\left(x^{2 i} y: i \in \mathbb{N}\right), \quad m_{4}:=\left(x^{2 i+2} y: i \in \mathbb{N}\right) .
$$

Then $m_{1} \otimes m_{2}-m_{3} \otimes m_{4}$ lies in $\operatorname{Ker}\left(\beta_{2}\right)-\{0\}$.

Proof. For $i, j \in \mathbb{N}$, both $m_{1} \otimes m_{2}$ and $m_{3} \otimes m_{4}$ have $x^{2 i+2 j+2} y^{2}$ as their $(i, j)$ th component. Thus $m_{1} \otimes m_{2}-m_{3} \otimes m_{4} \in \operatorname{Ker}\left(\beta_{2}\right)$.

Let $I$ denote the ideal of $R$ generated by $\left\{x^{2 i} y, x^{2 i} y^{2}: i \in \mathbb{N}\right\}$, and let $R^{\prime}:=R / I$. Then $R^{\prime}$ is the commutative augmented $Q$-ring with augmentation ideal of square zero and $Q$-basis $X:=\left\{x^{2 i+1} y: i \in \mathbb{N}\right\}$. In $\prod_{\mathbb{N}} R^{\prime}$, let $m:=\left(x^{2 i+1} y: i \in \mathbb{N}\right)$. By Proposition 6.7] in $\left(\prod_{\mathbb{N}} R^{\prime}\right)^{\otimes 2}, m \otimes m \neq 0$. The natural map $\left(\prod_{\mathbb{N}} R\right)^{\otimes 2} \rightarrow\left(\prod_{\mathbb{N}} R^{\prime}\right)^{\otimes 2}$ carries $m_{1} \otimes m_{2}-m_{3} \otimes m_{4}$ to $(m \otimes m)-(0 \otimes 0) \neq 0$. Hence, $m_{1} \otimes m_{2}-m_{3} \otimes m_{4} \neq 0$.

\section{Acknowledgments}

The first-named author was partially supported by DGI MICIIN MTM2011-28992-C02-01, and by the Comissionat per Universitats i Recerca de la Generalitat de Catalunya.

The second-named author was partially supported by Spain's Ministerio de Ciencia e Innovación through Project MTM2011-25955.

We are very grateful to George Bergman for generously contributing the examples of Section 6 and suggesting many improvements of various earlier versions.

We are also grateful to Dolors Herbera for providing much useful information for Section 4.

\section{References}

[1] Pere Ara and Warren Dicks, Universal localizations embedded in power-series rings, Forum Math. 19 (2007), 365-378.

[2] Hyman Bass, Finitistic dimension and a homological generalization of semi-primary rings, Trans. Amer. Math. Soc. 95 (1960), 466-488.

[3] George M. Bergman, Ordering coproducts of groups and semigroups, J. Algebra 133 (1990), 313-339.

[4] Vasily C. Cateforis, On regular self-injective rings, Pacific J. Math. 30 (1969), 39-45.

[5] Stephen U. Chase, Direct products of modules, Trans. Amer. Math. Soc. 97 (1960), 457-473.

[6] P. M. Cohn, On the free product of associative rings, Math. Z. 71 1959, 380-398. 
[7] P. M. Cohn, Free ideal rings, J. Algebra 1 (1964), 47-69.

[8] P. M. Cohn, Free rings and their relations. Second edition, LMS Monographs 19, Academic Press, London, 1985. xxii+588 pp.

[9] S.H. Cox and R.L. Pendleton, Rings for which certain flat modules are projective, Trans. Amer. Math. Soc. 150 (1970), 139-156.

[10] B. Eckmann and A. Schopf, Über injektive Moduln, Arch. Math. 4 (1953), 75-78.

[11] Ralph H. Fox, Free differential calculus. I Derivation in the free group ring, Ann. of Math. (2) $\mathbf{5 7}$ (1953), 547-560.

[12] K. R. Goodearl, Distributing tensor product over direct product, Pacific J. Math. 43 (1972), $107-110$.

[13] Dolors Herbera and Jan Trlifaj, Almost free modules and Mittag-Leffler conditions, Adv. Math. 229 (2012), 3436-3467.

[14] C. U. Jensen, Some cardinality questions for flat modules and coherence, J. Algebra 12 (1969), 231-241.

[15] S. Jøndrup, On finitely generated flat modules III, Math. Scand. 29 (1971), 206-210.

[16] Marsha Finkel Jones, Flatness and f-projectivity of torsion-free modules and injective modules, pp. 94-116 in: Advances in noncommutative ring theory. Proc. Twelfth George H. Hudson Symp., SUNY, Plattsburgh NY, 1981, (ed. Patrick J. Fleury), Lecture Notes in Math. 951, Springer-Verlag, Berlin, 1982. iii+142 pp.

[17] Irving Kaplansky, Projective modules, Ann. of Math. 68 (1958), 372-377.

[18] Irving Kaplansky, On the dimension of modules and algebras, $X$ A right hereditary ring which is not left hereditary, Nagoya Math. J. 13 (1958), 85-88.

[19] Shigeru Kobayashi, A note on regular self-injective rings, Osaka J. Math. 21 (1984), 679-682.

[20] Helmut Lenzing, Endlich präsentierbare Moduln, Arch. Math. 20 (1969), 262-266.

[21] Wilhelm Magnus, Beziehungen zwischen Gruppen und Idealen in einem speziellen Ring, Math. Ann. 111 (1935), 259-280.

[22] B. L. Osofsky, Rings all of whose finitely generated modules are injective, Pacific J. Math. 14 (1964), 645-650.

[23] Joseph J. Rotman, An introduction to homological algebra, Pure and applied mathematics 89, Academic Press, New York, 1979. xi+376 pp.

[24] I. I. Sahaev, The projectivity of finitely generated flat modules, Mat.Ž. 6 (1965), 564-573.

Departament de Matemàtiques, Universitat Autònoma de Barcelona, E-08193 Bellaterra (Barcelona), Spain

E-mail addresses: para@mat.uab.cat dicks@mat.uab.cat

Home page: http://mat.uab.cat/ dicks/ 